\title{
ARTÍCULOS
}

\section{FACTORES PSICOSOCIALES ASOCIADOS AL CONFLICTO ENTRE MENORES EN EL CONTEXTO ESCOLAR*}

\author{
Aura Alicia Cardozo-Rusinque ${ }^{1}$ (D) \\ Marina Begoña Martínez-González ${ }^{1}$ (D) \\ Adriana Angélica De La Peña-Leiva ${ }^{1}$ (D) \\ INÍRIDA AVEDAÑo-VILLA ${ }^{1}$ (D) \\ Tito José Crissien-Borrero ${ }^{1}$ (D)
}

\begin{abstract}
RESUMEN: Se exploró la relación entre factores psicosociales del contexto y el afrontamiento de conflictos en edad escolar. Una muestra de 378 niños (158 niños y 220 niñas) residentes en Barranquilla (Colombia), respondieron a un videojuego que simula situaciones de conflicto. El sexo fue determinante en el afrontamiento y en la expectativa sobre los pares y los adultos como sancionadores, neutrales o legitimadores de la violencia. El barrio y la televisión fueron los escenarios donde los niños observan más violencia, pero inciden en una mayor utilización del diálogo, lo que puede indicar modelos positivos de solución de conflictos más eficientes que los presentados por la familia. Se sugiere considerar los programas televisivos y los acuerdos comunitarios como complementos en la educación para la sana convivencia desde la infancia.
\end{abstract}

Palabras claves: Estrategias de solución de conflictos. Infancia. Conflicto escolar. Violencia escolar.

\section{FATORES PSICOSSOCIAIS ASSOCIADOS AO CONFLITO INFANTIL NA ESCOLA}

RESUMO: Esta pesquisa explorou a relação entre fatores psicossociais contextuais e como $s$ crianças lidam com possíveis conflitos na escola. Uma amostra de 378 crianças (158 meninos e 220 meninas) de Barranquilla (Colômbia) reagiu a um videogame que simula situaçôes de conflito. Seu sexo foi decisivo nas estratégias de enfrentamento para lidar com tais conflitos e foi relacionado às expectativas das crianças em relação a seus colegas e adultos como punidores, neutros ou legitimadores da

\footnotetext{
*Este artículo es producto de la investigación denominada "Observatorio de Convivencia Escolar para el Caribe Colombiano: Escenario de Paz. Fase I”, identificado con el código INV. 150-01-004-12, financiado por la Universidad de la Costa.

${ }^{1}$ Universidad de la Costa - Barranquilla, Atlántico, Colombia. E-mails: acardozo@cuc.edu.co; mmartine21@cuc.edu.co; adelapena@cuc.edu.co; iavendano@cuc.edu.co; rectoria@cuc.edu.co DOI: 10.1590/ES0101-73302019189140
} 
violência. A vizinhança e a televisão foram os cenários onde as crianças observam mais violência, mas implicam um maior uso do diálogo, o que pode indicar modelos positivos de resoluçáo de conflitos mais eficientes do que os apresentados por suas famílias. Sugere-se considerar os programas de televisão como um complemento da educaçáo para uma convivência saudável.

Palavras-chave: Estratégias de resolução de conflitos. Infância. Conflito acadêmico. Violência escolar

\section{PSYCHOSOCIAL FACTORS ASSOCIATED TO CHILDREN'S CONFLICT AT SCHOOL}

ABSTRACT: This research explored the relationship between contextual psychosocial factors and how infants deal with conflicts that could appear at school. A sample of 378 children (158 boys and 220 girls) from Barranquilla (Colombia) responded to a video game that simulates conflict situations. Their gender was decisive in coping strategies to deal with conflicts and it was related to the expectation that children have about peers and adults as punishers, neutral or legitimators of violence. Neighborhood and television were the scenarios where children observe more violence, but they imply a greater use of dialogue, which may indicate positive models of conflict resolution more efficient than those presented by their families. It is suggested to consider television programs as a complement in education for a healthy coexistence.

Keywords: Conflict-solving strategies. Childhood. Scholar conflict. Scholar violence.

\section{Introducción}

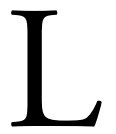

a edad escolar, es el período de la vida en el que niños desarrollan una mayor independencia, interacción social e identificación con sus pares adultos lo que refuerza su autoafirmación (RUBIN et al., 1998). En la niñez surge la necesidad de pertenencia a un grupo, lo que lleva al incremento de las amistades, pero también la competencia y las agresiones (ROSE \& RUDOLPH, 2006) que abren paso a formas sostenidas de maltrato en el ámbito escolar. La violencia en esta edad emerge dentro del aprendizaje de las normas sociales, que se interiorizan a través del juego y las interacciones diarias con pares y adultos (RUBIN et al., 1998), cuyo manejo desde su rol de mediadores, será crucial para favorecer una sana adaptación social o para consolidar creencias justificadoras de la violencia (MARTÍNEZ-GONZÁLEZ et al., 2016; FERNÁNDEZ VILLANUEVA, 2009). 
Múltiples estudios señalan que en el proceso de conformación del grupo de amigos, la agresión física entre varones aparece como medio de establecimiento de jerarquías sociales (p.e., CAMPO DÍAZ et al., 2012; CARD et al., 2008; ARCHER, 2004), mientras que las nińas, responden de manera prosocial ante los conflictos (HOPMEYER \& ASHER, 1997; CHUNG \& ASHER, 1996) debido a un mayor desarrollo de la empatía (HARTMANN et al., 2015). Sin embargo, se ha encontrado que las niñas usan formas de agresión encubierta, usando mecanismos de exclusión social (BENENSON, 2013) y aunque suelen lograr relaciones más profundas y vinculantes (RUBIN et al., 1998), también son más frágiles y menos duraderas (BENENSON \& CHRISTAKOS, 2003), presentando más dificultades que los varones para resolver los conflictos, que experimentan de forma intensa en términos emocionales que las lleva a tardar más en reconciliarse (BENENSON et al., 2014). Al parecer estas situaciones responden a prácticas de crianza que juzgan más negativamente la agresión entre las niñas y promueven una mayor necesidad de aprobación por parte de terceros (MARTÍNEZ-GONZÁLEZ, 2017).

Entre los elementos de atribución en la manera cómo los niños afrontan los conflictos, se destacan tres situaciones (FERNÁNDEZ VILLANUEVA, 2009): la percepción sobre quién inicia el conflicto, la relación de poder entre las partes implicadas y la percepción de no tener otra alternativa distinta a la agresión para dar solución a la situación. Los niños y niñas que legitiman el uso de la violencia, atribuyen el comienzo del conflicto al otro; justifican el uso de la violencia en una ventaja basada en la autoridad que puede darse por la edad, el género, mayor fuerza, etc., o atribuyen el uso de la violencia como último recurso cuando se percibe una desventaja en el conflicto. Por su parte, los niños que no legitiman la violencia, recurren a estrategias basadas en sus propios recursos, en su grupo de pares o a un adulto como mediadores ante el conflicto, cediendo ante el oponente o huyendo de la situación (FERNÁNDEZ VILLANUEVA, 2009).

Las dinámicas particulares del manejo de los conflictos en la infancia están relacionadas con el proceso de socialización, donde las expectativas de los adultos sobre el aprendizaje de normas sociales y las relaciones de género y de pares, determina en gran medida los recursos sociales que ellos desarrollan para interactuar (MARTÍNEZ-GONZÁLEZ, 2017).

El conflicto presente en el contexto escolar es una expresión de las dinámicas familiares, comunitarias, sociales e institucionales (VARELA-GARAY et al., 2013) que moldean creencias justificadoras del uso de la violencia en el proceso de socialización y crianza (FERNÁNDEZ VILLANUEVA, 2009; MARTÍNEZ-GONZÁLEZ et al., 2016).

Los nińos forman parte de una estructura familiar, donde pueden encontrarse actitudes asociadas a la imposición de significados y violencia simbólica en el ejercicio de la autoridad (ESTÉVEZ-LÓPEZ et al., 2008), de aplicar las sanciones (CONDE FLORES, 2014), de normalizar, legitimar o promover la vio- 
lencia (MARTÍNEZ-GONZÁLEZ et al., 2016), todo lo cual afecta la calidad del clima y de los patrones de comunicación en la familia (BUELGA et al., 2017).

El estudio del afrontamiento de conflictos interpersonales cotidianos en la infancia, ha permitido identificar, que los niños y niñas recurren a justificaciones sobre el uso de la violencia (FERNÁNDEZ VILLANUEVA, 2009); pueden ser menos empáticos y más competitivos cuando han crecido bajo la utilización de estilos parentales autoritarios (MARTÍNEZ-GONZÁLEZ et al., 2016; CUERVO MARTINEZ, 2010; ÁLVAREZ-GALLEGO, 2010; MESTRE ESCRIVÁ et al., 2001).

La familia es fundamental en el ajuste psicosocial de sus miembros, cuando se tiene buena comunicación e implicación en la formación de los hijos. Junto a ella, la escuela se constituye en un escenario fundamental para la convivencia o para la manifestación de la violencia escolar (ESTÉVEZ-LÓPEZ et al., 2008), que se genera en las interacciones entre los miembros de la comunidad educativa en el ejercicio de sus roles (D'ANGELO \& FERNÁNDEZ, 2011). Muchas veces los procesos institucionales favorecen la aparición de conflictos caracterizados por el poder asimétrico y la sensación de desamparo en los afectados, dado el distanciamiento entre las lógicas adultas y las necesidades de los estudiantes (GARCÉS-MONTOYA, 2006). Los docentes en ocasiones se perciben con pocas herramientas efectivas para enfrentar esta situación, presentan confusión en la definición de funciones y responsabilidades entre padres y maestros y viven una discrepancia de valores y normas con las que fueron criados (GARCÍA-SÁNCHEZ, 2008).

Ahora bien, la violencia escolar, también es expresión del conflicto social más amplio, reflejando desigualdades económicas y socioculturales del contexto (CAJIGAS et al., 2004). Los estudiantes reproducen en el ámbito escolar la violencia que viven en el hogar o en la calle (GARCÍA-SÁNCHEZ, 2003; CONDE FLORES, 2014). Estos suelen estar enmarcados por situaciones de pobreza, falta de oportunidades, desconfianza en las fuerzas del orden, deserción escolar, baja remuneración del trabajo no calificado, violencia intrafamiliar, pandillas, entre otras (COSTA \& ROMERO, 2009), que promueven relaciones conflictivas desde temprana edad (HERRERA-MENDOZA \& RICO-BALLESTEROS, 2014).

El deterioro de la apreciación del barrio y de la identidad social de sus integrantes, genera la pérdida de mecanismos de control social y el surgimiento de la anomia y la indiferencia, debido a que las personas ya no comparten valores de solidaridad y convivencia comunitaria, no hay cohesión, ni redes sociales fuertes (COSTA \& ROMERO, 2009; ROMERO-VILLADÓNIGA, 2016). Estas situaciones constituyen un factor determinante para el surgimiento de pandillas y el incremento de la violencia escolar (RAMÍREZ-LÓPEZ \& RODRÍGUEZ, 2013), dificultando la gestión positiva de los conflictos (DÍAZ-BETTER, 2016), generando una cultura de la calle en la que pierde valor la ciudadanía, dando lugar 
a actitudes desafiantes, menosprecio por las normas y pérdida del sentido de la importancia del otro (CONDE FLORES, 2014).

La violencia presente en escenarios de socialización afectiva como son la familia, la escuela y la comunidad, deben atenderse junto con las relaciones entre los actores sociales implicados, que pueden asumirse como el lazo para la transmisión de valores y pautas de comportamiento a ser apropiados por los nińos y los jóvenes (GARCÍA-SÁNCHEZ, 2008).

Los aprendizajes sobre las formas para establecer relaciones a partir de vínculos sociales directos, también se ven determinados por los contenidos que se transmiten a través de los medios de comunicación presentes en todas las dinámicas de la vida cotidiana (MARTÍNEZ-GONZÁLEZ et al., 2011). Los niños acceden contenidos violentos a través de estos medios, generando preocupación sobre la relación entre lo que transmiten y las conductas agresivas en la infancia (ERON et al., 1974). Esto, considerando que los medios masivos recrean escenas que simulan la realidad, establecen pautas para la construcción de la identidad y divulgan valores y contravalores (MARÍN-DÍAZ \& SOLÍS, 2017; BELLONI, 2004).

Ahora bien, el reconocimiento de la influencia que tienen los medios masivos en la educación, ha generado una transformación en los contenidos para consumo infantil, dando lugar a reflexiones sobre los derechos humanos, la equidad de género y la inclusión. Al respecto se ha encontrado que los nińos que observan programas con contenido prosocial, presentan menos prejuicios hacia personas de los grupos étnicos observados en ellos y otros evidencian una mayor apropiación de la equidad de género (HOPKINS \& WEISBERG, 2017). En consecuencia, se puede considerar que estos programas pueden funcionar como una herramienta para el cambio social desde la infancia favoreciendo la empatía, el desarrollo de una visión crítica y la apropiación de VALORES (DE LEEUW et al., 2015; PADILLA-WALKER et al., 2015; MARÍN-DÍAZ \& SOLÍS, 2017).

La presente investigación analiza el afrontamiento de los conflictos en edad escolar, teniendo en cuenta tres aspectos: las decisiones de resolución de los conflictos, la expectativa que tienen los niños sobre sus pares y sobre los adultos en el conflicto y los escenarios de exposición a la violencia, para lo cual se ha tenido en cuenta la familia, el barrio y la televisión con relación a su incidencia en el proceso de socialización y el curso de las relaciones interpersonales desde la infancia.

\section{Metodología}

Se realizó una investigación de alcance explicativo con múltiples covariables (BONO-CABRÉ, 2012). Se propusieron diseños factoriales para establecer la relación entre variables independientes y dependientes. El primer grupo de hipótesis buscaba diferencias relacionadas con el sexo en el afrontamiento del con- 
flicto, la percepción de pares y adultos y los escenarios de exposición a contenido violento. El segundo grupo identifica los escenarios donde los niños observan la violencia y su relación con el afrontamiento.

Participaron 378 estudiantes entre los 7 y los 10 años (158 niños y 220 nińas), vinculados a escuelas del sector público de la ciudad de Barranquilla, Colombia. Los niños respondieron al videojuego "Leyendas de Almar ${ }^{\circledR}$ " (MARTÍNEZ-GONZÁLEZ et al., 2014), que plantea tres situaciones de conflicto:

- Igualdad: evalúa el uso de la violencia como legítima defensa ante un oponente percibido como semejante;

- Ventaja: evalúa la tendencia a usar la violencia por autoridad, cuando se percibe superioridad sobre el oponente;

- Desventaja: que evalúa la tendencia al uso de la violencia como anticipación a una amenaza.

Los datos se procesaron a partir del análisis de frecuencias, la prueba $\mathrm{Chi}^{2}$ y t de Student para las pruebas de hipótesis.

\section{Resultados}

Se encontró una relación significativa entre el sexo y el tipo de decisión tomada para afrontar el conflicto, en caso de igualdad $(\mathrm{p}=0,002)$ y en desventaja $(p=0,013)$.

En la situación de igualdad (Tabla 1), considerando muestras independientes, se encuentra que los niños atacan en mayor porcentaje $(36,1 \%)$ que las niñas $(19,1 \%)$, usando la violencia por sí mismos. Sin embargo, las distribuciones no son diferentes cuando piden ayuda a otros para atacar al oponente. La opción de dialogar muestra mayor tendencia en las mujeres (50\%) que en los hombres (38\%), igual que la opción de huida (12,7 y 8,2\%, respectivamente). Decisiones que implican la participación de terceros, muestran que los niños piden más ayuda para calmar al oponente $(9,5 \%)$ que las nińas $(5,5 \%)$, mientras que delegar a otros el fin de la tarea interrumpida se evidencia más en las mujeres $(8,6 \%)$ que en los hombres $(4,4 \%)$.

Esto se replica en la situación de desventaja (Tabla 2). Los niños atacan más que las niñas (22,8 y 10,5\% para cada caso) cuando usan la violencia por sí mismos, mientras que las distribuciones no difieren cuando piden ayuda a otros para este fin. En esta situación la tendencia a dialogar es mucho más marcada tanto en hombres como en mujeres ( 62,7 y $68,6 \%$ respectivamente). La opción de huir fue la menos escogida, aunque se observa mayor tendencia en las niñas que 


\section{Tabla 1}

Afrontamiento del conflicto en situación de igualdad según el sexo del participante.

\begin{tabular}{|c|c|c|c|c|c|c|c|c|}
\hline \multirow{3}{*}{ Sexo } & & \multicolumn{6}{|c|}{ Decisiones } & \multirow[b]{3}{*}{ Total } \\
\hline & & \multicolumn{3}{|c|}{ Alternativas personales } & \multicolumn{3}{|c|}{ Alternativas con terceros } & \\
\hline & & $\begin{array}{l}\text { Atacar al } \\
\text { oponente }\end{array}$ & Dialogar & Huir & $\begin{array}{l}\text { Que otros } \\
\text { ayuden a } \\
\text { calmar al } \\
\text { oponente }\end{array}$ & $\begin{array}{c}\text { Que otros } \\
\text { ataquen al } \\
\text { oponente }\end{array}$ & $\begin{array}{c}\text { Que otros } \\
\text { ayuden al } \\
\text { logro del } \\
\text { objetivo }\end{array}$ & \\
\hline \multirow{2}{*}{ Femenino } & $\mathrm{n}$ & 42,00 & 110,00 & 28,00 & 12,00 & 9,00 & 19,00 & 220,0 \\
\hline & $\%$ & 19,00 & 50,0 & 12,7 & 5,5 & 4,1 & 8,6 & 100,0 \\
\hline \multirow{2}{*}{ Masculino } & $\mathrm{n}$ & 57,00 & 60,00 & 13,00 & 15,00 & 6,00 & 7,00 & 158,0 \\
\hline & $\%$ & 36,1 & 38,0 & 8,2 & 9,5 & 3,8 & 4,4 & 100,0 \\
\hline \multirow{2}{*}{ Total } & $\mathrm{n}$ & 99,00 & 170,00 & 41,00 & 27,00 & 15,00 & 26,00 & 378,0 \\
\hline & $\%$ & 26,2 & 45,0 & 10,8 & 7,1 & 4,0 & 6,9 & 100,0 \\
\hline
\end{tabular}

Prueba de Chi cuadrado

\begin{tabular}{c|c|c|c}
\hline \multicolumn{2}{c|}{ Valor } & gl & p \\
\hline$\chi^{2}$ & 19,29 & 5 & 0,002 \\
\hline $\mathrm{N}$ & 378 & & \\
\hline
\end{tabular}

Tabla 2

Afrontamiento del conflicto en situación de desventaja según el sexo del participante.

\begin{tabular}{|c|c|c|c|c|c|c|c|c|}
\hline \multirow[b]{3}{*}{ Sexo } & & \multicolumn{6}{|c|}{ Decisiones } & \multirow[b]{3}{*}{ Total } \\
\hline & & \multicolumn{3}{|c|}{ Alternativas personales } & \multicolumn{3}{|c|}{ Alternativas con terceros } & \\
\hline & & $\begin{array}{l}\text { Atacar al } \\
\text { oponente }\end{array}$ & Dialogar & Huir & $\begin{array}{l}\text { Que otros } \\
\text { ayuden a } \\
\text { calmar al } \\
\text { oponente }\end{array}$ & $\begin{array}{c}\text { Que otros } \\
\text { ataquen al } \\
\text { oponente }\end{array}$ & $\begin{array}{c}\text { Que otros } \\
\text { ayuden al } \\
\text { logro del } \\
\text { objetivo }\end{array}$ & \\
\hline \multirow{2}{*}{ Femenino } & $\mathrm{n}$ & 23,00 & 151,00 & 17,00 & 10,00 & 5,00 & 14,00 & 220,0 \\
\hline & $\%$ & 10,5 & 68,6 & 7,7 & 4,5 & 2,3 & 6,4 & 100,0 \\
\hline \multirow{2}{*}{ Masculino } & $\mathrm{n}$ & 36,00 & 99,00 & 5,00 & 4,00 & 2,00 & 12,00 & 158,0 \\
\hline & $\%$ & 22,8 & 62,7 & 3,2 & 2,5 & 1,3 & 7,6 & 100,0 \\
\hline \multirow{2}{*}{ Total } & $\mathrm{n}$ & 59,00 & 250,00 & 22,00 & 14,00 & 7,00 & 26,00 & 378,0 \\
\hline & $\%$ & 15,6 & 66,1 & 5,8 & 3,7 & 1,9 & 6,9 & 100,0 \\
\hline
\end{tabular}

Prueba de Chi cuadrado

\begin{tabular}{c|c|c|c}
\hline \multicolumn{2}{c|}{ Valor } & gl & p \\
\hline$\chi^{2}$ & 14,46 & 5 & 0,013 \\
\hline $\mathrm{N}$ & 378 & & \\
\hline
\end{tabular}


en los niños $(7,7$ y 3,2\%). Las opciones con participación de terceros fueron poco seleccionadas por ambos grupos, encontrándose una mayor tendencia en niños a pedir a otros que terminen la tarea interrumpida por el conflicto $(7,6$ y $6,4 \%)$.

Los resultados encontrados con relación a la expectativa sobre los pares y los adultos en las tres situaciones (Tablas 3, 4 y 5) muestran diferencias entre niños y niñas (en general $\mathrm{p}<0.001$ ).

En situación de igualdad se encontró que el $82 \%$ de los participantes que espera sanción de los pares también la espera de los adultos, con idéntica distribución en niñas y niños (100\%). Se encuentra que el 50\% de las niñas espera sanción de los adultos y neutralidad de los pares, mientras que el 20\% de ellas espera sanción de los adultos y apoyo de los pares. En el caso de los varones, el $66,7 \%$ espera sanción de los adultos y neutralidad de los pares, frente a un 50\% que espera sanción de adultos y legitimación de pares. En general, el 50\% de los participantes esperó legitimación de pares y adultos. Esto ocurre en el $70 \%$ de las niñas y en un 33\% de los varones. La expectativa de legitimidad por parte de los adultos y neutralidad de los pares se encuentra solo en las nińas, en una proporción del $25 \%$. La expectativa de neutralidad en pares y adultos, se encuentra en el $30 \%$ de los participantes, evidenciándose en el $25 \%$ de las nińas y el $33,3 \%$ de los varones. El 10\% de las niñas y el 16\% de los varones esperan neutralidad de los adultos y legitimación de los pares.

En situación de ventaja, se encuentra que el 100\% de los participantes que tiene expectativa de sanción por parte de los pares también la tiene de los adultos. El 50\% de las mujeres espera sanción de los adultos y neutralidad de los pares, caso que no se presenta en los varones; el $75 \%$ de las nińas y el 33,3\% de los niños, espera sanción de los adultos y legitimidad de los pares. La legitimación esperada tanto de pares y adultos aparece en el $40 \%$ de los participantes, siendo $25 \%$ para las niñas y $50 \%$ de los niños. Estos últimos evidencian en un 33,3\% una expectativa de legitimidad de los adultos y neutralidad de los pares, que no se presenta en niñas. La expectativa de neutralidad total (pares y adultos) se manifiesta en el $60 \%$ de la muestra, correspondiendo al 50\% de las nińas y al 66,7\% de los nińos. Solo los varones esperan neutralidad de los adultos y legitimación de los pares (16,7\%).

También en situación de desventaja cuando la sanción que se espera de los pares también se espera de los adultos (100\%). La sanción de adultos y neutralidad de los pares aparece en el $28,6 \%$ de las niñas y el $40 \%$ de los niños; la sanción de adultos y legitimidad de pares se presenta en el 50\% de las niñas y en el 30\% de los niños. La legitimidad percibida tanto en pares como adultos se presenta en el $61,5 \%$ de los participantes, siendo presente en un $50 \%$ de las niñas y $65 \%$ de los niños. El 28,6\% de las nińas espera legitimación de los adultos y neutralidad de los pares, lo cual no se manifiesta en los niños. La expectativa de neutralidad de pares y adultos, coincide en el $50 \%$ de los participantes, con un $42,9 \%$ de las niñas y un 


\section{Tabla 3}

Expectativa sobre los pares y los adultos ante el uso de la violencia en situación de igualdad según el sexo del participante.

\begin{tabular}{|c|c|c|c|c|c|c|}
\hline \multirow{2}{*}{ Sexo } & \multirow{2}{*}{\multicolumn{2}{|c|}{$\begin{array}{c}\text { Expectativa } \\
\text { sobre los pares }\end{array}$}} & \multicolumn{3}{|c|}{ Expectativa sobre los adultos } & \multirow{3}{*}{$\begin{array}{c}\text { Total } \\
37,00\end{array}$} \\
\hline & & & Sanciona & Neutro & Legitima & \\
\hline \multirow{8}{*}{ Femenino } & \multirow{2}{*}{ Sanciona } & $\mathrm{n}$ & 37,00 & 0,00 & 0,00 & \\
\hline & & $\%$ & 100,0 & 0,0 & 0,0 & 100,0 \\
\hline & \multirow{2}{*}{ Neutro } & $\mathrm{n}$ & 2,00 & 1,00 & 1,00 & 4,00 \\
\hline & & $\%$ & 50,0 & 25,0 & 25,0 & 100,0 \\
\hline & \multirow{2}{*}{ Legitima } & $\mathrm{n}$ & 2,00 & 1,00 & 7,00 & 10,00 \\
\hline & & $\%$ & 20,0 & 10,0 & 70,0 & 100,0 \\
\hline & \multirow{2}{*}{ Total } & $\mathrm{n}$ & 41,00 & 2,00 & 8,00 & 51,00 \\
\hline & & $\%$ & 80,4 & 3,9 & 15,7 & 100,0 \\
\hline \multirow{8}{*}{ Masculino } & \multirow{2}{*}{ Sanciona } & $\mathrm{n}$ & 45,00 & 0,00 & 0,00 & 45,00 \\
\hline & & $\%$ & 100,0 & 0,0 & 0,0 & 100,0 \\
\hline & \multirow{2}{*}{ Neutro } & $\mathrm{n}$ & 4,00 & 2,00 & 0,00 & 6,00 \\
\hline & & $\%$ & 66,7 & 33,3 & 0,0 & 100,0 \\
\hline & \multirow{2}{*}{ Legitima } & $\mathrm{n}$ & 6,00 & 2,00 & 4,00 & 12,00 \\
\hline & & $\%$ & 50,0 & 16,7 & 33,3 & 100,0 \\
\hline & \multirow{2}{*}{ Total } & $\mathrm{n}$ & 55,00 & 4,00 & 4,00 & 63,00 \\
\hline & & $\%$ & 87,3 & 6,3 & 6,3 & 100,0 \\
\hline \multirow{8}{*}{ Total } & \multirow{2}{*}{ Sanciona } & $\mathrm{n}$ & 82,00 & 0,00 & 0,00 & 82,00 \\
\hline & & $\%$ & 100,0 & 0,0 & 0,0 & 100,0 \\
\hline & \multirow{2}{*}{ Neutral } & $\mathrm{n}$ & 6,00 & 3,00 & 1,00 & 10,00 \\
\hline & & $\%$ & 60,0 & 30,0 & 10,0 & 100,0 \\
\hline & \multirow{2}{*}{ Legitima } & $\mathrm{n}$ & 8,00 & 3,00 & 11,00 & 22,00 \\
\hline & & $\%$ & 36,4 & 13,6 & 50,0 & 100,0 \\
\hline & \multirow{2}{*}{ Total } & $\mathrm{n}$ & 96,00 & 6,00 & 12,00 & 114,00 \\
\hline & & $\%$ & 84,2 & 5,3 & 10,5 & 100,0 \\
\hline
\end{tabular}

Prueba de Chi cuadrado

\begin{tabular}{l|c|c|c|c}
\hline Sexo & \multicolumn{2}{|c|}{ Valor } & gl & p \\
\hline \multirow{2}{*}{ Femenino } & $\chi^{2}$ & 38,52 & \multirow{2}{*}{4} & $<, 001$ \\
\cline { 2 - 3 } & $\mathrm{N}$ & 51 & & \\
\hline \multirow{2}{*}{ Masculino } & $\chi^{2}$ & 31,79 & \multirow{2}{*}{4} & $<, 001$ \\
\cline { 2 - 3 } & $\mathrm{N}$ & 63 & & \\
\hline \multirow{2}{*}{ Total } & $\chi^{2}$ & 69,18 & \multirow{2}{*}{4} & \multirow{2}{*}{$<, 001$} \\
\cline { 2 - 3 } & $\mathrm{N}$ & 114 & & \\
\hline
\end{tabular}


Tabla 4

Expectativa sobre los pares y los adultos ante el uso de la violencia en situación de ventaja según el sexo del participante.

\begin{tabular}{|c|c|c|c|c|c|c|}
\hline \multirow{2}{*}{ Sexo } & \multirow{2}{*}{\multicolumn{2}{|c|}{$\begin{array}{c}\text { Expectativa sobre } \\
\text { los pares }\end{array}$}} & \multicolumn{3}{|c|}{ Expectativa sobre los adultos } & \multirow{3}{*}{$\begin{array}{c}\text { Total } \\
14,00\end{array}$} \\
\hline & & & Sanciona & Neutro & Legitima & \\
\hline \multirow{8}{*}{ Femenino } & \multirow{2}{*}{ Sanciona } & $\mathrm{n}$ & 14,00 & 0,00 & 0,00 & \\
\hline & & $\%$ & 100,0 & 0,0 & 0,0 & 100,0 \\
\hline & \multirow{2}{*}{ Neutro } & $\mathrm{n}$ & 1,00 & 1,00 & 0,00 & 2,00 \\
\hline & & $\%$ & 50,0 & 50,0 & 0,0 & 100,0 \\
\hline & \multirow{2}{*}{ Legitima } & $\mathrm{n}$ & 3,00 & 0,00 & 1,00 & 4,00 \\
\hline & & $\%$ & 75,0 & 0,0 & 25,0 & 100,0 \\
\hline & \multirow{2}{*}{ Total } & $\mathrm{n}$ & 18,00 & 1,00 & 1,00 & 20,00 \\
\hline & & $\%$ & 90,0 & 5,0 & 5,0 & 100,0 \\
\hline \multirow{8}{*}{ Masculino } & \multirow{2}{*}{ Sanciona } & $\mathrm{n}$ & 21,00 & 0,00 & 0,00 & 21,00 \\
\hline & & $\%$ & 100,0 & 0,0 & 0,0 & 100,0 \\
\hline & \multirow{2}{*}{ Neutro } & $\mathrm{n}$ & 0,00 & 2,00 & 1,00 & 3,00 \\
\hline & & $\%$ & 0,0 & 66,7 & 33,3 & 100,0 \\
\hline & \multirow{2}{*}{ Legitima } & $\mathrm{n}$ & 2,00 & 1,00 & 3,00 & 6,00 \\
\hline & & $\%$ & 33,3 & 16,7 & 50,0 & 100,0 \\
\hline & \multirow{2}{*}{ Total } & $\mathrm{n}$ & 23,00 & 3,00 & 4,00 & 30,00 \\
\hline & & $\%$ & 76,7 & 10,0 & 13,3 & 100,0 \\
\hline \multirow{8}{*}{ Total } & \multirow{2}{*}{ Sanciona } & $\mathrm{n}$ & 35,00 & 0,00 & 0,00 & 35,00 \\
\hline & & $\%$ & 100,0 & 0,0 & 0,0 & 100,0 \\
\hline & \multirow{2}{*}{ Neutral } & $\mathrm{n}$ & 1,00 & 3,00 & 1,00 & 5,00 \\
\hline & & $\%$ & 20,0 & 60,0 & 20,0 & 100,0 \\
\hline & \multirow{2}{*}{ Legitima } & $\mathrm{n}$ & 5,00 & 1,00 & 4,00 & 10,00 \\
\hline & & $\%$ & 50,0 & 10,0 & 40,0 & 100,0 \\
\hline & \multirow{2}{*}{ Total } & $\mathrm{n}$ & 41,00 & 4,00 & 5,00 & 50,00 \\
\hline & & $\%$ & 82,0 & 8,0 & 10,0 & 100,0 \\
\hline
\end{tabular}

Prueba de Chi cuadrado

\begin{tabular}{l|c|c|c|c}
\hline Sexo & \multicolumn{2}{|c|}{ Valor } & gl & p \\
\hline \multirow{2}{*}{ Femenino } & $\chi^{2}$ & 13,61 & \multirow{2}{*}{4} & \multirow{2}{*}{0,009} \\
\cline { 2 - 3 } & $\mathrm{N}$ & 20 & & \\
\hline \multirow{2}{*}{ Masculino } & $\chi^{2}$ & 27,01 & \multirow{2}{*}{4} & $<, 001$ \\
\cline { 2 - 3 } & $\mathrm{N}$ & 30 & & \\
\hline \multirow{2}{*}{ Total } & $\chi^{2}$ & 37,73 & \multirow{2}{*}{4} & \multirow{2}{*}{$<, 001$} \\
\cline { 2 - 3 } & $\mathrm{N}$ & 50 & & \\
\hline
\end{tabular}




\section{Tabla 5}

Expectativa sobre los pares y los adultos ante el uso de la violencia en situación de desventaja según el sexo del participante.

\begin{tabular}{|c|c|c|c|c|c|c|}
\hline \multirow{2}{*}{ Sexo } & \multirow{2}{*}{\multicolumn{2}{|c|}{$\begin{array}{c}\text { Expectativa sobre } \\
\text { los pares }\end{array}$}} & \multicolumn{3}{|c|}{ Expectativa sobre los adultos } & \multirow{3}{*}{$\begin{array}{c}\text { Total } \\
15,00\end{array}$} \\
\hline & & & Sanciona & Neutro & Legitima & \\
\hline \multirow{8}{*}{ Femenino } & \multirow{2}{*}{ Sanciona } & $\mathrm{n}$ & 15,00 & 0,00 & 0,00 & \\
\hline & & $\%$ & 100,0 & 0,0 & 0,0 & 100,0 \\
\hline & \multirow{2}{*}{ Neutro } & $\mathrm{n}$ & 2,00 & 3,00 & 2,00 & 7,00 \\
\hline & & $\%$ & 28,6 & 42,9 & 28,6 & 100,0 \\
\hline & \multirow{2}{*}{ Legitima } & $\mathrm{n}$ & 3,00 & 0,00 & 3,00 & 6,00 \\
\hline & & $\%$ & 50,0 & 0,0 & 50,0 & 100,0 \\
\hline & \multirow{2}{*}{ Total } & $\mathrm{n}$ & 20,00 & 3,00 & 5,00 & 28,00 \\
\hline & & $\%$ & 71,4 & 10,7 & 17,9 & 100,0 \\
\hline \multirow{8}{*}{ Masculino } & \multirow{2}{*}{ Sanciona } & $\mathrm{n}$ & 13,00 & 0,00 & 0,00 & 13,00 \\
\hline & & $\%$ & 100,0 & 0,0 & 0,0 & 100,0 \\
\hline & \multirow{2}{*}{ Neutro } & $\mathrm{n}$ & 2,00 & 3,00 & 0,00 & 5,00 \\
\hline & & $\%$ & 40,0 & 60,0 & 0,0 & 100,0 \\
\hline & \multirow{2}{*}{ Legitima } & $\mathrm{n}$ & 6,00 & 1,00 & 13,00 & 20,00 \\
\hline & & $\%$ & 30,0 & 5,0 & 65,0 & 100,0 \\
\hline & \multirow{2}{*}{ Total } & $\mathrm{n}$ & 21,00 & 4,00 & 13,00 & 38,00 \\
\hline & & $\%$ & 55,3 & 10,5 & 34,2 & 100,0 \\
\hline \multirow{8}{*}{ Total } & \multirow{2}{*}{ Sanciona } & $\mathrm{n}$ & 28,00 & 0,00 & 0,00 & 28,00 \\
\hline & & $\%$ & 100,0 & 0,0 & 0,0 & 100,0 \\
\hline & \multirow{2}{*}{ Neutral } & $\mathrm{n}$ & 4,00 & 6,00 & 2,00 & 12,00 \\
\hline & & $\%$ & 33,3 & 50,0 & 16,7 & 100,0 \\
\hline & \multirow{2}{*}{ Legitima } & $\mathrm{n}$ & 9,00 & 1,00 & 16,00 & 26,00 \\
\hline & & $\%$ & 34,6 & 3,8 & 61,5 & 100,0 \\
\hline & \multirow{2}{*}{ Total } & $\mathrm{n}$ & 41,00 & 7,00 & 18,00 & 66,00 \\
\hline & & $\%$ & 62,1 & 10,6 & 27,3 & 100,0 \\
\hline
\end{tabular}

Prueba de Chi cuadrado

\begin{tabular}{l|c|c|c|c}
\hline Sexo & \multicolumn{2}{|c|}{ Valor } & gl & p \\
\hline \multirow{2}{*}{ Femenino } & $\chi^{2}$ & 19,50 & \multirow{2}{*}{4} & $<, 001$ \\
\cline { 2 - 3 } & $\mathrm{N}$ & 28 & & \\
\hline \multirow{2}{*}{ Masculino } & $\chi^{2}$ & 32,50 & \multirow{2}{*}{4} & $<, 001$ \\
\cline { 2 - 3 } & $\mathrm{N}$ & 38 & & \\
\hline \multirow{2}{*}{ Total } & $\chi^{2}$ & 52,21 & \multirow{2}{*}{4} & $<, 001$ \\
\cline { 2 - 3 } & $\mathrm{N}$ & 66 & & \\
\hline
\end{tabular}


$60 \%$ de los niños. La expectativa de neutralidad de los adultos y legitimación por parte de los pares solo se presenta en varones (5\%).

Los participantes señalaron observar más situaciones de violencia en el barrio $(\mathrm{n}=142)$ y la televisión $(\mathrm{n}=123)$, y en menor medida todas las opciones $(\mathrm{n}=63)$ y la casa $(\mathrm{n}=48)$. El análisis del afrontamiento de los conflictos según los escenarios de exposición a contenido violento (Tabla 6), mostró relación significativa solo en la situación de conflicto en ventaja $(\mathrm{p}=0.021)$.

La mayor proporción de participantes frente a la opción de atacar por sí mismos se encontró en los participantes que reportan observar violencia en todos los escenarios (19\%) seguidos de quienes la observan en la casa $(18,8 \%)$. La decisión de dialogar, se encuentra con una mayor tendencia en los niños y nińas que afirman ver violencia en la televisión (76,4\%), seguidos de quienes la observan en el barrio $(76,1 \%)$, encontrándose una amplia diferencia entre quienes la ven en el hogar $(64,6 \%)$ y quienes señalan todos los escenarios $(55,6 \%)$. La decisión de huir se presentó más en quienes afirman ver la violencia en todos los escenarios $(15,9 \%)$ y en el barrio $(8,5 \%)$.

\section{Tabla 6}

Afrontamiento de los conflictos en situación de ventaja según el escenario de exposición a la violencia.

\begin{tabular}{l|c|c|c|c|c|c|c|c}
\hline \multirow{2}{*}{ Escenarios } & \multicolumn{7}{c}{ Decisiones } & \multirow{2}{*}{ Total } \\
\cline { 3 - 10 } \multicolumn{2}{c|}{} & Atacar & $\begin{array}{c}\text { Ataquen } \\
\text { al otro }\end{array}$ & $\begin{array}{c}\text { Ayuden } \\
\text { a buscar }\end{array}$ & $\begin{array}{c}\text { Ayuden a } \\
\text { calmarlo }\end{array}$ & Dialogar & Huir & \\
\hline \multirow{2}{*}{ Barrio } & $\mathrm{n}$ & 12,00 & 3,00 & 4,00 & 3,00 & 108,00 & 12,00 & 142,00 \\
\cline { 2 - 10 } & $\%$ & 8,5 & 2,1 & 2,8 & 2,1 & 76,1 & 8,5 & 100,0 \\
\hline \multirow{2}{*}{ Casa } & $\mathrm{n}$ & 9,00 & 2,00 & 3,00 & 1,00 & 31,00 & 2,00 & 48,00 \\
\cline { 2 - 10 } & $\%$ & 18,8 & 4,2 & 6,3 & 2,1 & 64,6 & 4,2 & 100,0 \\
\hline \multirow{2}{*}{ Televisión } & $\mathrm{n}$ & 7,00 & 3,00 & 7,00 & 6,00 & 94,00 & 6,00 & 123,00 \\
\cline { 2 - 10 } & $\%$ & 5,7 & 2,4 & 5,7 & 4,9 & 76,4 & 4,9 & 100,0 \\
\hline \multirow{2}{*}{ Todas } & $\mathrm{n}$ & 12,00 & 1,00 & 1,00 & 4,00 & 35,00 & 10,00 & 63,00 \\
\cline { 2 - 9 } & $\%$ & 19,0 & 1,6 & 1,6 & 6,3 & 55,6 & 15,9 & 100,0 \\
\hline \multirow{2}{*}{ Total } & $\mathrm{n}$ & 40,00 & 9,00 & 15,00 & 14,00 & 268,00 & 30,00 & 376,00 \\
\cline { 2 - 9 } & $\%$ & 10,6 & 2,4 & 4,0 & 3,7 & 71,3 & 8,0 & 100,0 \\
\hline
\end{tabular}

Prueba de Chi cuadrado

\begin{tabular}{l|c|c|c}
\hline \multicolumn{2}{|l|}{ Valor } & gl & $\mathbf{p}$ \\
\hline$\chi^{2}$ & 28,04 & & \\
\cline { 1 - 1 } $\mathrm{N}$ & 376 & \multirow{2}{*}{15} & 0,021 \\
\cline { 1 - 2 } Perdidos & 2 & & \\
\hline
\end{tabular}




\section{Discusión}

El manejo del conflicto y la violencia escolar, requiere del reconocimiento de las formas como los niños y nińas toman posturas frente a estos, con relación a cómo los perciben y cómo se perciben a sí mismos y a sus capacidades para gestionarlos.

Las diferencias en la manera cómo los niños y niñas asumen el conflicto, se ha asociado a elementos socioculturales que llevan a los hombres a usar más la violencia por sí mismos o con ayuda de otros (GIMÉNEZ-GARCÍA et al., 2014; MUNOZ-TINOCO et al., 2008). Este patrón sociocultural, por otra parte, ha delegado a la mujer el cuidado y bienestar de los que le rodean (RUIZ PINTO et al., 2013), lo que demanda el desarrollo de habilidades para la consecución de apoyo de terceros que legitimen y refuercen su autoridad (CHAVES RUBIO, 2002; MAÑERU, 2007). Esto explicaría la tendencia de las niñas a optar por el diálogo a la finalización de su objetivo, implicando la existencia de habilidades estereotipadas hacia lo considerado como femenino (MESTRE ESCRIVÁ et al., 1998) con relación a estrategias de mitigación del conflicto que implican la evitación (MILLER et al., 1986). Los varones por su parte, delegan la finalización de la tarea cuando se perciben en desventaja, lo que ha sido observado en niños que buscan apoyo cuando se perciben con menos recursos (MUÑOZ-TINOCO et al., 2008). La autopercepción de niños y niñas respecto a sus habilidades sociales y recursos para la solución de conflictos, ha sido relacionada con la percepción que tienen de sus padres, señalando un patrón de autodesprecio, baja autonomía e incertidumbre sobre el amor en los varones, mientras que la autoculpa y una menor autoafirmación son más frecuente en las niñas (YBRANDT \& ARMELIUS, 2010).

En esta investigación las expectativas de sanción de los pares y los adultos frente al uso de la violencia, dan cuenta de la capacidad de los infantes para reconocer el quebrantamiento a las normas sociales que esta opción implica. Las niñas, experimentan mayor temor a la sanción social de los adultos quienes las evalúan como menos violentas (DEAUX \& MAJOR, 1987; CARD et al., 2008), lo que incrementa su necesidad de aprobación social, la preocupación por el estado de sus amistades, temor al abandono (ROSE \& RUDOLPH, 2006; HENRICH et al., 2001) y a la evaluación de sus pares (RUDOLPH \& CONLEY, 2005; STORCH et al., 2003). Pese a que los niños también señalaron una expectativa de sanción de pares y adultos, también es notorio que presentaran una mayor expectativa de neutralidad o de legitimación de los adultos (MARTÍNEZ-GONZÁLEZ, 2017). Esto corresponde a una crianza en el que suelen incitarlos a usar la fuerza e imponerse para ganar respeto y autoafirmarse en las situaciones de conflicto, como parte de la construcción de lo masculino (URRÁ, 2003; GINI \& POZZOLI, 2006). 
En lo referente a la percepción de violencia en los distintos escenarios de socialización, se encuentra que la televisión se ha convertido en una fuente de soluciones alternativas al conflicto, mientras que el manejo dado por los adultos en el hogar, muestra pautas poco adaptativas para su afrontamiento.

Muchas han sido las críticas que se han hecho a los contenidos televisivos por su incidencia en la generación de conductas disruptivas desde la niñez. Sin embargo, la televisión contemporánea comienza a encontrarse como un escenario cuyos contenidos infantiles, priorizan aspectos como el diálogo, la inclusión de género, diversidad étnica, entre otras, aportando a la socialización positiva (HOPKINS \& WEISBERG, 2017). Los programas infantiles hoy hacen un reconocimiento y legitimación de la diversidad y la asertividad, de manera que los medios de comunicación, películas infantiles y videojuegos pueden ser una fuente importante de formación en valores para los niños. El logro de estos propósitos hace necesario el acompañamiento a los nińos por parte de los adultos desde la familia o en la escuela, de tal manera que generen reflexiones sobre los mensajes que se promueven en las plataformas transmedia. Aunque la educación ha avanzado en la incorporación de las TIC como facilitadoras de la apropiación de conceptos académicos, no se ha profundizado en las posibilidades que ofrecen para la formación en habilidades sociales y para la vida, que en general siguen enseñándose desde un enfoque tradicional. Los resultados de este estudio suman a los hallazgos relacionados con el potencial existente en las TIC como herramienta pedagógica aplicable a la educación para mejorar aspectos como la convivencia, constituyéndose en una estrategia de solución de conflictos, y generación de conductas prosociales en la edad escolar (AVENDAÑO-VILLA et al., 2015).

Ahora bien, también llama la atención la tendencia de optar por el diálogo de aquellos niños que señalaron ver violencia en el barrio. Las relaciones de identidad y cooperación presentes en la vida de comunidad, pueden ser un escenario propicio para la construcción de redes de apoyo y de interrelación psicológicamente significativos (MAYA-JARIEGO, 2009) que sirvan de protección ante fenómenos como la aparición de pandillas juveniles. Las formas de resistencia civil y comunitaria ante manifestaciones de la violencia en el territorio, podrían aportar a la apropiación de formas pacíficas y al potencial creativo en la resolución de conflictos desde temprana edad (NARVÁEZ, 2013).

Así las cosas, son los padres quienes enfrentan el desafío de generar mejores prácticas frente al afrontamiento de los conflictos. Esta investigación coincide con la literatura que señala a la violencia en el hogar como incidente en la aparición de conductas poco adaptativas desde la infancia. Las familias que enfrentan inestabilidad, pautas transgeneracionales de violencia, escasa formación en habilidades sociales (PULIDO et al., 2013), enfrentan grandes dificultades para 
la formación en convivencia y para el establecimiento de modelos positivos en la resolución y afrontamiento de conflictos. Contrariamente, si a edades tempranas los niños cuentan con un acompañamiento paterno favorable para la resolución de conflictos, mostrarán una mayor variedad de mecanismos de ajuste cuando los tenga que enfrentar (RICAUD-DROISY \& ZAOUCHE-GAUDRON, 2003). Las relaciones establecidas con los padres como modelos de interacción con los demás, favorecerá el desarrollo de la autonomía, la integración social y la apertura a relaciones interpersonales amplias.

Las nuevas generaciones tienen el reto de aprender a vivir con otros en medio de la desconfianza y la desilusión sobre las instituciones y una cada vez más impersonal sociedad de masas (TOURAINE \& PONS, 1997). En este escenario se ve inmersa la escuela, que debe superar la creencia generalizada de que la educación cumple básicamente un papel de formar para la vida laboral y productiva (RESTREPO, 2006), descuidando la preparación para el cambio, la realización personal (BAUMAN, 2003) y la formación ciudadana. Tanto la familia como la escuela deben comprender que la convivencia en la contemporaneidad implica nuevas formas de relación, de construir los afectos, las amistades y las relaciones amorosas, de vivir en la familia, en la escuela; así como también de entender la ética, los valores, de construir sociedad dando prioridad a la dignidad humana y al respeto por los derechos humanos (ARELLANO, 2007). La complejidad en la que se desenvuelve el ser humano, demanda la construcción de procesos deliberados hacia la formación para la convivencia, más allá de las normas tradicionales (CARDOZO, 2017), particularmente en los escenarios que conforman los niveles micro sociales (familia-escuela) y meso-sociales (barrio-comunidad). Como se ha señalado éstos resultan fundamentales para posibilitar la solución pacífica de los conflictos, consolidar la formación moral, establecer redes de apoyo que den identidad, sean modelo legítimo de convivencia y aporten a la posibilidad real de construir una mejor sociedad.

\section{Referencias}

ALVARÁN-LÓPEZ, S.M. et al. Estudio exploratorio para el análisis de los efectos psicosociales de la violencia en la infancia colombiana. Àgora de Salut, v. 1, n. 1, p. 547-559, 2016. http://dx.doi.org/10.6035/AgoraSalut.2015.1.45

ÁLVAREZ-GALLEGO, M. M. Prácticas educativas parentales: autoridad familiar, incidencia en el comportamiento agresivo infantil. Revista Virtual Universidad Católica del Norte, n. 31, p. 253-273, 2010. Disponible en: <http://www.redalyc.org/articulo. oa?id=194214587011>. Acceso en el: 13 mayo 2017.

ARCHER, J. Sex differences in aggression in realworld settings: A meta-analytic review. Review of General Psychology, v. 8, n. 4, p. 291-322, 2004. http://dx.doi. org/10.1037/1089-2680.8.4.291 
ARELLANO, N. La violencia escolar y la provención del conflicto. Orbis: Revista de Ciencias Humanas, v. 3, n. 7, p. 23-45, 2007. Disponible en: <http://ojs.revistaorbis.org. ve/index.php/orbis/article/view/269>. Acceso en el: 3 ago. 2017.

AVENDAÑO-VILLA, I.; CORTÉS-PEÑA, O. F.; GUERRERO-CUENTAS, H. Competencias sociales y tecnologías de la información y la comunicación como factores asociados al desempeño en estudiantes de básica primaria con experiencia de desplazamiento forzado. Revista Diversitas - Perspectivas en Psicología, v. 11, n. 1, p. 13-36, 2015. Disponible en: <http:/www.scielo.org.co/pdf/dpp/v11n1/v11n1a02.pdf >. Acceso en el: 17 nov. 2017.

BAUMAN, Z. Modernidad líquida. México: Fondo de Cultura Económica, 2003.

BELLONI, M. L. Infância, máquinas e violência. Educação \& Sociedade, v. 25, n. 87, p. 575-598, 2004. http://dx.doi.org/10.1590/S0101-73302004000200012

BENENSON, J. F. The development of human female competition: allies and adversaries. Philosophical Transactions of Royal Society, v. 368, n. 1631, 2013. http://dx.doi. org/10.1098/rstb.2013.0079

BENENSON, J. F.; CHRISTAKOS, A. The greater fragility of females' versus males' closest same-sex friendships. Child Development, v. 74, n. 4, p. 1123-1129, 2003. http:// dx.doi.org/10.1111/1467-8624.00596

BENENSON, J.F. et al. Human males appear more prepared than females to resolve conflicts with same-sex peers. Human Nature, v. 25, n. 2, p. 251-268, 2014. http:// dx.doi.org/10.1007/s12110-014-9198-z

BONO-CABRÉ, R. Diseños cuasi-experimentales y longitudinales. Barcelona, 2012. Disponible en: <http://hdl.handle.net/2445/30783 > . Acceso en el: 20 sept. 2017.

BUELGA, S.; MARTÍNEZ-FERRER, B.; CAVA, M. J. Differences in family climate and family communication among cyberbullies, cybervictims, and cyber bully-victims in adolescents. Computers in Human Behavior, v. 76, p. 164-173, 2017. https://doi. org/10.1016/j.chb.2017.07.017

CAJIGAS DE SEGREDO, N. et al. Escala de agresión entre pares para adolescentes y principales resultados [Bullying scale for adolescents and main results]. Acción Psicológica, v. 3, n. 3, p. 173-186, 2004. https://doi.org/10.5944/ap.3.3.511

CAMPO DÍAZ, M. S.; ESCORCIA ESCORCIA, D. E.; HERNÁNDEZ ARROYAVE, C. Modelo teórico comprensivo de la vivencia del bullying en víctimas y agresores a partir del juego de rol (RPG): un estudio de niños y niñas entre 7 y 9 ańos, en la ciudad de Barranquilla. Tesis (Pregrado en Psicología) - Facultad de Psicología, Universidad de la Costa, Barranquilla, 2012. Disponible en: <http://hdl.handle.net/11323/91>. Acceso en el: 17 nov. 2017.

CARD, N. A.; STUCKY, B. D.; SAWALANI, G. M.; LITTLE, T. D. Direct and indirect aggression during childhood and adolescence: A meta-analytic review of gender differences, intercorrelations, and relations to maladjustment. Child development, v. 79, n. 5, p. 1185-1229, 2008. https://doi.org/10.1111/j.1467-8624.2008.01184.x 
CHAVES RUBIO, C. Autoridad y mediación femenina como práctica de la paz. Duoda: Revista d'estudis Feministes, n. 23, p. 65-81, 2002.

CHUNG, T.; ASHER, S. R. Children's goals and strategies in peer conflict situations. Merrill-Palmer Quarterly, v. 42, n. 1, p. 125-147, 1996. Disponible en: <http://www. jstor.org/stable/23090523>. Acceso en el: 17 nov. 2017.

CONDE FLORES, S. La violencia y la cultura de la calle entran a la escuela. Sinéctica Revista Electrónica de Educación, n. 42, 2014. Disponible en: <http://www.sinectica. iteso.mx/articulo/?id=42>. Acceso en el: 13 nov. 2017.

COSTA, G.; ROMERO, C. ¿Qué hacer con las pandillas? Lima: Ciudad Nuestra, 2009.

CUERVO MARTINEZ, A. Pautas de crianza y desarrollo socioafectivo en la infancia. Diversitas: Perspectivas en Psicología, v. 6, n. 1, p. 111-121, 2010. Disponible en: <http://www.redalyc.org/articulo.oa?id=67916261009>. Acceso en el: 23 mayo 2017.

D’ANGELO, L.; FERNÁNDEZ, D. Clima, conflicto y violencia en la escuela. Fondo de las Naciones Unidas para la Infancia (UNICEF)/Facultad Latinoamericana de Ciencias Sociales (FLACSO), 2011. Disponible en: < https://issuu.com/unicef-tacro/docs/clima conflicto violencia escuelas $>$. Acceso en el: 1 nov. 2017.

DEAUX, K.; MAJOR, B. Putting gender into context: An interactive model of genderrelated behavior. Psychological Review, v. 94, n. 3, p. 369-389, jul. 1987. http://dx.doi. org/10.1037/0033-295X.94.3.369

DE LEEUW, R.N. et al. The impact of prosocial television news on children's prosocial behavior: An experimental study in the Netherlands. Journal of Children and Media, v. 9, n. 4, p. 419-434, 2015. https://doi.org/10.1080/17482798.2015.1089297

DÍAZ-BETTER, S. Imaginarios de docentes sobre convivencia escolar: reflexiones desde un núcleo de educación social (NES). En: SIMPOSIO INTERNACIONAL, 3., y COLOQUIO REGIONAL DE INVESTIGACIÓN EDUCATIVA Y PEDAGÓGICA, 4., 2016. Anales [...]. 2016.

ERON, L. D. et al. How learning conditions in early childhood - including mass media - relate to aggression in late adolescence. American Journal of Orthopsychiatry, v. 44, n. 3, p. 412-423, 1974. http://dx.doi.org/10.1111/j.1939-0025.1974.tb00894.x

ESTÉVEZ-LÓPEZ, E.; PÉREZ, S. M.; OCHOA, G. M.; RUIZ, D. M. Adolescent aggression: Effects of gender and family and school environments. Journal of Adolescence, v. 31, n. 4, p. 433-450, 2008. https://doi.org/10.1016/j.adolescence.2007.09.007

FERNÁNDEZ VILLANUEVA, I. Justificación y legitimación de la violencia en la infancia. Un estudio sobre la legitimación social de las agresiones en los conflictos cotidianos entre menores. 2009. Tesis (Doctorado en Psicología) - Facultad de Psicología, Universidad Complutense de Madrid, Madrid, 2009. Disponible en: < $\underline{\text { http://eprints.ucm. }}$ es/8436/>. Acceso en el: 15 ago. 2017.

GARCÉS-MONTOYA, Á. Juventud y escuela: percepciones y estereotipos que rondan el espacio escolar. Última Década, v. 14, n. 24, p. 61-77, 2006. http://dx.doi.org/10.4067/ $\underline{\text { S0718-22362006000100004 }}$ 
GARCÍA-SÁNCHEZ, B. Familia, escuela y barrio: un contexto para la comprensión de la violencia escolar. Revista Colombiana de Educación, v. 55, p. 108-124, 2008. Disponible en: <http://www.redalyc.org/articulo.oa?id=413635249006>. Acceso en el: 7 abr. 2017.

GARCÍA-SÁNCHEZ, B. Los núcleos de educación familiar y la prevención de las violencias difusas en contextos educativos. Revista Colombiana de Psicología, v. 12, p. 49-59, 2003. Disponible en: <http://www.redalyc.org/html/804/80401205/>. Acceso en el: 7 abr. 2017.

GIMÉNEZ-GARCÍA, C. et al. Roles de género y agresividad en la adolescencia. International Journal of Developmental and Educational Psychology, v. 2, n. 1, p. 373-382, 2014. http://dx.doi.org/10.17060/ijodaep.2014.n1.v2.452

GINI, G.; POZZOLI, T. The role of masculinity in children's bullying. Sex Roles, v. 54 n. 7-8, 585-588, 2006. http://dx.doi.org/10.1007/s11199-006-9015-1

HARTMANN, T.; MÖLLER, I.; KRAUSE, C. Factors underlying male and female use of violent video games. New Media \& Society, v. 17, n. 11, p. 1777-1794, 2015. http:// dx.doi.org/10.1177/1461444814533067

HENRICH, C. et al. Levels of interpersonal concerns and social functioning in early adolescent boys and girls. Journal of Personality Assessment, v. 76, n. 1, p. 48-67, 2001. https://doi.org/10.1207/S15327752JPA7601 3

HERRERA-MENDOZA, K.; RICO-BALLESTEROS, R. Clima escolar como elemento fundamental de la convivencia en la escuela. Revista Escenarios, v. 12, n. 2, 2014. http:// dx.doi.org/10.15665/esc.v12i2.311

HOPKINS, E.J.; WEISBERG, D.S. The youngest readers' dilemma: A review of children's learning from fictional sources. Developmental Review, v. 43, p. 48-70, 2017. https:// doi.org/10.1016/j.dr.2016.11.001

HOPMEYER, A.; ASHER, S. R. Children's responses to peer conflicts involving a rights infraction. Merrill-Palmer Quarterly, v. 43, n. 2, p. 235-254, 1997. Disponible en: <http://www.jstor.org/stable/23092490 >. Acceso en el: 24 mayo 2017.

MAÑERU, A. La práctica de la autoridad en las relaciones. Crítica, v. 943, p. 55-59, 2007.

MARÍN-DÍAZ, V.; SOLÍS, C. Los valores transmitidos por las mujeres en las películas Disney. Revista CS, v. 23, p. 37-55, 2017. http://dx.doi.org/10.18046/recs.i23.2296

MARTÍNEZ-GONZÁLEZ, M. B. Estudios sobre mujer. Cartagena de Indias: Sello Editorial Tecnológico de Comfenalco, 2017.

MARTÍNEZ-GONZÁLEZ, M. B. et al. Leyendas de Almar ${ }^{\circledR} 2014$.

MARTÍNEZ-GONZÁLEZ, M. B.; FRÍAS, W. P.; SOLANO, D. Impacto de los medios masivos de comunicación en la dinámica familiar. Cultura Educación y Sociedad, v. 2, n. 1, p. 111-118, 2011. Disponible en: <http://revistascientificas.cuc.edu.co/index.php/ culturaeducacionysociedad/article/view/940/pdf 160>. Acceso en el: 19 jun. 2017. 
MARTÍNEZ-GONZÁLEZ, M. B.; ROBLES-HAYDAR, C. A.; AMAR-AMAR, J. J.; CRESPO-ROMERO, F. A. Crianza y desconexión moral en infantes: Su relación en una comunidad vulnerable de Barranquilla. Revista Latinoamericana de Ciencias Sociales, Niñez y Juventud, v. 14, n. 1, p. 315-330, 2016. http://dx.doi. org/10.11600/1692715x.14121011214

MAYA-JERIEGO, I. Sentido de comunidad y potenciación comunitaria. Miríada: Investigación en Ciencias Sociales, v. 2, n. 3, p. 69-109, 2009. Disponible en: $<\underline{\text { http:// }}$ p3.usal.edu.ar/index.php/miriada/article/view/399>. Acceso en el: 23 mayo 2017.

MESTRE ESCRIVÁ, M. V.; DELGADO, E. P.; GARCÍA, P. S.; VILAR, M. M. Diferencias de género en la empatía y su relación con el pensamiento moral y el altruismo. Anales de la Revista de Psicología General y Aplicada, v. 3, n. 1, p. 1-16, 1998. Disponible en: $<$ https://dialnet.unirioja.es/servlet/articulo?codigo=300817> . Acceso en el: 23 mayo 2017.

MESTRE ESCRIVÁ, M. V.; GARCÍA, P. S.; PORCAR, A. M. T.; DÍEZ, I. Estilos de crianza y desarrollo prosocial de los hijos. Revista de Psicología General y Aplicada, v. 54, n. 4, p. 691-703, 2001. Disponible en: <https://dialnet.unirioja.es/ servlet/articulo?codigo=2364995>. Acceso en el: 30 oct. 2017.

MILLER, P. M.; DANAHER, D. L.; FORBES, D. Sex-related strategies for coping with interpersonal conflict in children aged five and seven. Developmental Psychology, v. 22, n. 4, p. 543-548, 1986. http://dx.doi.org/10.1037/0012-1649.22.4.543

MUÑOZ-TINOCO, M. V.; JIMÉNEZ-LAGARES, I.; MORENO RODRIGUÉZ, M. C. Reputación conductual y género en la adolescencia. Anales de Psicología, v. 24, n. 2, p. 334-340, 2008. Disponible en: <http://www.redalyc.org/html/167/16711589017/>. Acceso en el: 23 mayo 2017.

NARVÁEZ, J. H. Violencia barrial, la tensión social como crisis humanitaria. Tesis Psicológica: Revista de la Facultad de Psicología, v. 8, n. 1, p. 56-67, 2013.

PADILLA-WALKER, L. M.; COYNE, S. M.; COLLIER, K. M.; NIELSON, M. G. Longitudinal relations between prosocial television content and adolescents' prosocial and aggressive behavior: The mediating role of empathic concern and self-regulation. Developmental Psychology, v. 51, n. 9, p. 1317-1328, 2015. http:// dx.doi.org/10.1037/a0039488

PULIDO, S.; CASTRO-OSORIO, J.; PEÑA, M.; ARIZA-RAMÍREZ, D. P. Pautas, creencias y prácticas de crianza relacionadas con el castigo y su transmisión generacional. Revista Latinoamericana de Ciencias Sociales, Nińez y Juventud, v. 11, n. 1, p. 245 259, 2013. http://dx.doi.org/10.1037/a003948810.11600/1692715x.11116030912

RAMÍREZ-LÓPEZ, C. A.; ARCILA-RODRÍGUEZ, W. Violencia, conflicto y agresividad en el escenario escolar. Educación y Educadores, v. 16, n. 3, p. 6, 2013. Disponible en: $<$ https://dialnet.unirioja.es/servlet/articulo?codigo=5468350 > Acceso en el: 8 oct. 2017.

RESTREPO, J. C. Estándares básicos en competencias ciudadanas: una aproximación al problema de la formación ciudadana en Colombia. Papel Político, v. 11, n. 1, p. 137-176, 2006. Disponible en: <https://bit.ly/2Lsvhs1 >. Acceso en el: 17 ago. 2017. 
RICAUD-DROISY, H.; ZAOUCHE-GAUDRON, C. Interpersonal conflict resolution strategies in children: A father-child co-construction. European Journal of Psychology of Education, v. 18, n. 2, p. 157, 2003. https://doi.org/10.1007/BF03173482

ROMERO-VILLADÓNIGA, J. C. Mi gente, mi mundo. Una reflexión antropoeducativa en voz alta sobre el conflicto escolar. Antropología Experimental, v. 16, p. 431-441, 2016. https://dx.doi.org/10.17561/rae.v0i16.2210

ROSE, A. J.; RUDOLPH, K. D. A Review of sex differences in peer relationship processes: potential trade-offs for the emotional and behavioral development of girls and boys. Psychological Bulletin, v. 132, n. 1, p. 98-131, 2006. https://dx.doi.org/10.1037/0033$\underline{2909.132 .1 .98}$

RUBIN, K.; BUKOWSKI, W.; PARKER, J. Peer interactions, relationships, and groups. In: DAEMON, W.; EISEMBERG, N. (orgs.). Handbook of child psychology: social, emotional, and personality development. 5. ed. Nueva York: Wiley, 1998. v. 3. p. 619-700.

RUDOLPH, K.; CONLEY, C. The Socioemotional Costs and Benefits of Social-Evaluative Concerns: Do Girls Care Too Much? Journal of Personality, v. 73, n. 1, p. 115-138, 2005. https://doi.org/10.1111/j.1467-6494.2004.00306.x

RUIZPINTO, E.; GARCÍA PÉREZ, R.; REBOLLO, M.A. Relaciones de género de adolescentes en contextos educativos. Análisis de redes sociales con perspectiva de género. Profesorado. Revista de Currículum y Formación del Profesorado, v. 17, n. 1, p. 124-140, 2013.

TOURAINE, A.; PONS, H. ¿̨Podremos vivir juntos?: iguales y diferentes. Madrid: PPC, 1997. Disponible en: <https://bit.ly/2zMTurt>. Acceso en el: 17 sep. 2018.

URRÁ, J. Adolescencia y violencia. Tópicos y realidades. Revista de Estudios de Juventud, v. 62, p. 11-20, 2003. Disponible en: <http://www.injuve.es/sites/default/ files/62 completa.pdf\#page=9 $>$. Acceso en el: 22 ago. 2017.

VARELA-GARAY, R. M.; ÁVILA, E. M.; MARTÍNEZ, B. Violencia escolar: un análisis desde los diferentes contextos de interacción. Psychosocial Intervention, v. 22, n. 1, p. 25-32, 2013. https://dx.doi.org/10.5093/in2013a4

YBRANDT, H.; ARMELIUS, K. Adolescents' mental health and their images of self and parents. European Journal of Mental Health, v. 5, n. 1, p. 59, 2010. https://doi. org/10.1556/EJMH.5.2010.1.4

Recibido en: 14 de diciembre de 2017.

Acepto en: 19 de junio de 2018. 